\title{
Experimental determination of the induced activity in activation detectors of a complex geometric shape*
}

\author{
Renat F. Ibragimov ${ }^{1}$, Yakov A. Kokorev ${ }^{1}$, Anastasia P. Denisenko ${ }^{1}$, Elena V. Ryabeva ${ }^{1}$, \\ Valery T. Samosadny ${ }^{1}$, Hamza Hasnaoui ${ }^{1}$ \\ 1 National Research Nuclear University MEPhI, 31 Kashirskoe Hwy, 115409 Moscow, Russia \\ Corresponding author: Renat F. Ibragimov (rfibragimov@mephi.ru)
}

Academic editor: Yury Kazansky • Received 20 January 2020 • Accepted 17 August 2020 • Published 11 September 2020

Citation: Ibragimov RF, Kokorev YaA, Denisenko AP, Ryabeva EV, Samosadny VT, Hasnaoui H (2020) Experimental determination of the induced activity in activation detectors of a complex geometric shape. Nuclear Energy and Technology 6(3): 149-154. https:// doi.org/10.3897/nucet.6.57738

\begin{abstract}
The paper presents the results of experimental determination of the induced activity in copper-/aluminum-based activation detectors when irradiated with neutrons with energies of about $14 \mathrm{MeV}$. The activation detectors were squareshaped metal plates with a thickness from 1.0 to $1.5 \mathrm{~mm}$ and a side size about $5.0 \mathrm{~cm}$. These dimensions significantly exceed those of the detectors that are used in the research of high-intensity neutron fluxes. The detectors described in this work can be used for studying low-intensity neutron fluxes (with a flux density of up to $10^{6} \mathrm{n} / \mathrm{cm}^{2} \cdot \mathrm{s}$ ). It is shown that, when working with such detectors, it is possible to apply the usual methods for calculating the induced activity in thin activation detectors, with corrections that take into account the emerging features of the "neutron source - activation detector' and 'activation detector - secondary radiation detector' geometries. The effects of absorption of primary and secondary radiation by the detector substance are also revealed.

The Geant 4 tools were used for calculating the geometric factors and theoretical induced activity. The study confirms the applicability of such activation detectors for solving the problem of determining the yield of neutrons with energies of about $14 \mathrm{MeV}$ from a neutron generator target. The results of the experiments coincide, within the margin of error, with the results of simulations performed using the Geant 4 tools.
\end{abstract}

\section{Keywords}

Activation detectors, neutron radiation, induced activity, mathematical simulation, Geant4, geometric factor

Recommended for publication by the Organizing Committee of The $15^{\text {th }}$ International Youth Scientific and Practical Conference 'The Future of Nuclear Energy - AtomFuture 2019'

* Russian text published: Izvestiya vuzov. Yadernaya Energetika (ISSN 0204-3327), 2020, n. 2, pp. 156-167.

Copyright Ibragimov RF et al. This is an open access article distributed under the terms of the Creative Commons Attribution License (CC.BY 4.0), which permits unrestricted use, distribution, and reproduction in any medium, provided the original author and source are credited. 
Activation detectors exposed to neutron irradiation predominantly become emitters of gamma quanta or beta particles (electrons or positrons). The best studied neutron-detecting methods based on activation detectors are used in applications for analyzing the neutron field characteristics in the core of reactors or other nuclear facilities providing comparable values of neutron fluence (Andrukhovich et al. 2010, Jungmin 2018, Uddin et al. 2013). At the same time, for these methods of studying neutrons, it is sufficient to use small detectors (up to several millimeters in diameter and a fraction of a millimeter thick). Due to this feature, it becomes possible, in the subsequent analysis of the induced activity, to consider these detectors 'point-type' ones and neglect the corrections for self-absorption of gamma quanta or beta particles in their substance as well as ignore the sample thickness in terms of the probability of neutrons flying through the detector without interaction (Pierre et al. 2016, Noba 2019).

Activation detectors can also be used for studying low-intensity neutron fields, but this requires a significant increase in their thickness and area. The task of determining the activity in this case becomes much more complicated, since it is necessary to take into account the correction to the geometric detection efficiency of the gamma detector, the correction for the self-absorption of gamma quanta in the activation detector, and the effect of the detector thickness on the concentration of new isotopes formed in the material under the action of neutron radiation (Medkour Ishak-Boushaki 2012, Lebaron-Jacobs et al. 2008).

Low-intensity neutron sources include electrophysical devices (pulse- and continuous-type neutron generators), radioisotope sources and research pulsed facilities. In this case, the key advantages of activation detectors are the relatively small size of the activated samples (they do not introduce significant distortions in the neutron field), the key possibility of obtaining information on the integral neutron yield even in a very short pulse as well as the lack of sensitivity to accompanying gamma radiation (Vagena 2018). In addition to the direct benefits from studying the operating features of such neutron sources, acquiring the practical skills in examining the parameters of the above-mentioned neutron fields is a very important task in terms of preserving knowledge and implementing the possibility of high-quality training of young specialists for the nuclear industry.

This paper presents the results of the experimental determination of the induced activity in various samples of activation detectors, a description of the methods by which the calculations were made, and a comparison of the results of experimental measurements with the calculated values. The results of experimental measurements of the neutron yield from the neutron generator target are also shown. Activation detectors are metal plates $(\mathrm{Al}, \mathrm{Cu})$ with a natural isotopic composition, 1 to $1.5 \mathrm{~mm}$ thick, rectangular and square with sides from 3 to $5 \mathrm{~cm}$. As a neutron source, a portable neutron generator ING-07T manufactured by the FSUE Dukhov Automatics Research Institute (VNIIA) was used (Neutron Generators 2020) (Fig. 1). Each detector was irradiated in the field of neutrons with energies of about $14 \mathrm{MeV}$, after which the induced activity was recorded. The induced activity in these detectors was measured by analyzing the recorded pulse height spectrum of gamma radiation from these samples after their irradiation with a neutron flux. Secondary gamma quanta were detected using a coaxial detector made of highly pure germanium (HPGe), model Gem10P4, manufactured by ORTEC (Fig. 2).

Let us consider the problem of determining the induced activity using a sample of natural copper irradiated with neutrons with energies of about $14 \mathrm{MeV}$. This sample consists of $70 \%$ of ${ }^{63} \mathrm{Cu}$ and $30 \%$ of ${ }^{65} \mathrm{Cu}$. On these isotopes, $(n, 2 n)$ reactions mainly proceed to form ${ }^{62} \mathrm{Cu}$ and ${ }^{64} \mathrm{Cu}$ with half-lives of about 10 minutes and 13 hours, respectively. Both isotopes undergo positron decay, leading to the appearance of a $511 \mathrm{keV}$ line in the gamma spectrum. At the point, where the sample is located during irradiation, in addition to fast neutrons, there are scattered and moderated neutrons. This leads to the appearance of a small number of competing nuclear reactions, for example, the $(\mathrm{n}, \gamma)$ reaction on ${ }^{63} \mathrm{Cu}$, which also leads to the appearance of ${ }^{64} \mathrm{Cu}$. When the spectrum of the induced activity is recorded in the first 10-20 minutes, the contribution to the $511 \mathrm{keV}$ peak from ${ }^{64} \mathrm{Cu}$ can be neglected due to its long half-life as well as the small number of side $(n, \gamma)$ reactions.

Figure 3 shows the calculated energy spectra of neutrons at the location of the activation detector at the moment of irradiation. The calculations were carried out using the tools of the Geant4 software package (Allison 2016); a ready-made set of physics QGSP_BERT_HP was used as a physical list, allowing more accurate modeling of the behavior of neutrons with energies ranging from thermal ones to $20 \mathrm{MeV}$ in various substances. Two possible configurations were calculated: the presence and absence of biological protection in the form of $50 \mathrm{~cm}$ of borated polyethylene around the neutron generator. Figure 2 shows the visualization of the experimental model for irradiating the activation detectors with neutrons and recording the spectra of secondary gamma radiation with the HPGe detector.

The number of thermal neutrons appearing at the location of the activation detector is very small compared to the number of primary neutrons with energy of $14 \mathrm{MeV}$ (see Fig. 3). That is why it is possible to neglect both the reaction of capturing thermal neutrons by ${ }^{63} \mathrm{Cu}$ and the burnup of resulting ${ }^{62} \mathrm{Cu}$. Thus, the problem of determining the induced activity in a copper sample in this case is reduced to determining the activity of ${ }^{62} \mathrm{Cu}$ by the absolute method according to the number of detected annihilation gamma quanta. As a practical application of the described technique for calculating the induced activity in large activation detectors, the paper presents the result of measuring the neutron yield from the neutron generator target. The results of this experiment demonstrate the applicability of such detectors for studying low-intensity fields of neutron radiation. 


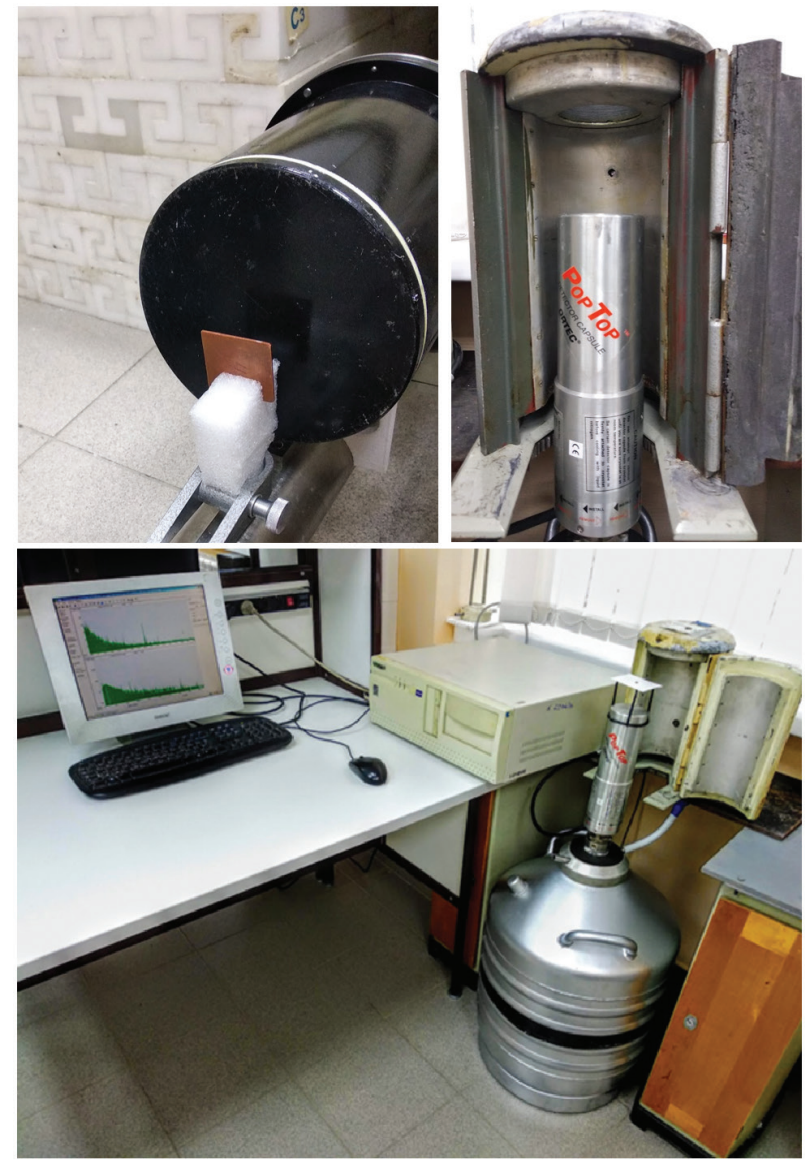

Figure 1. Appearance of the sample in the position for irradiation (in front of the end of the neutron generator, on the left), the detector in the protective house (open in the photo) and the workplace where the spectra were recorded.

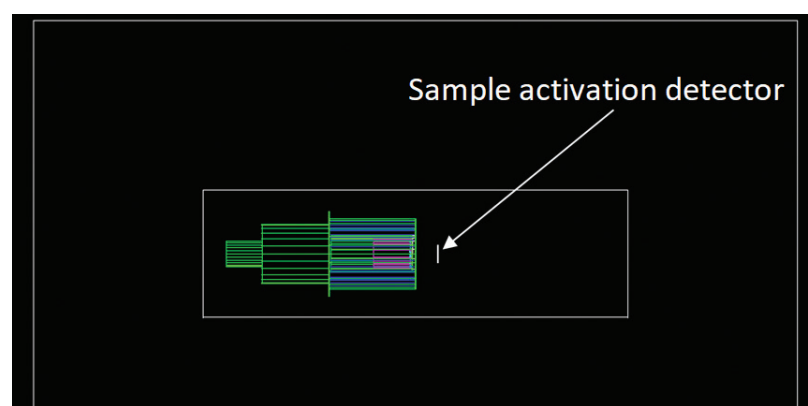

Biological protection (borated polyethylene, C3)

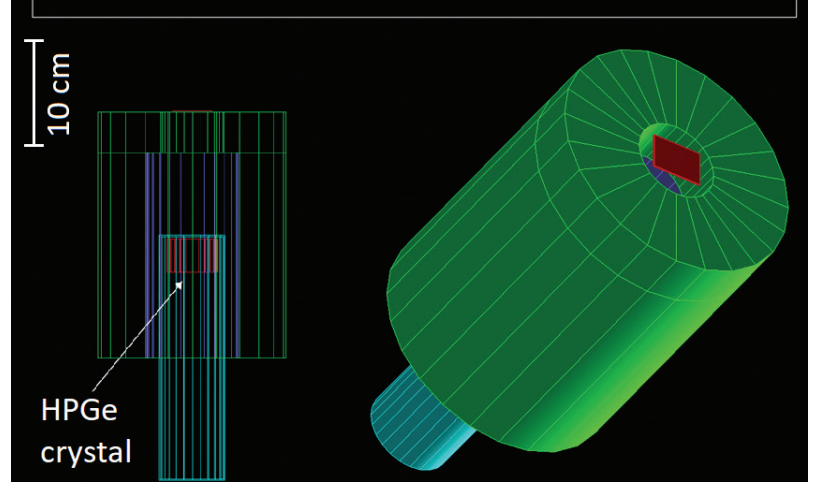

Figure 2. Visualizing the model of the experiment on irradiation (top) and recording the induced activity (calculating the geometric factor).
The activity of a point source of gamma radiation, the half-life of which is significantly longer than the activity measurement time, is described by the expression

$$
A=\frac{N_{\mathrm{det}}}{\varepsilon_{\mathrm{abs}} \cdot I \cdot t},
$$

where $N_{\text {det }}$ is the number of detected gamma quanta (integral number of pulses at the total absorption peak); $I$ is the quantum yield of the investigated line of gamma radiation in relative units; $t$ is the spectrum recording time; $\varepsilon_{a b s} \mathrm{~s}$ the absolute detection efficiency determined in relative units by the expression

$$
\varepsilon_{\text {abs }}=\frac{N_{\text {det }}}{N_{\text {emit }}},
$$

where $N_{\text {emit }}$ is the number of gamma quanta of a given energy emitted by the source into the full solid angle during the measurement of the activity. The dependence of the absolute efficiency on the energy of gamma quanta is determined by both the detector features and the measurement geometry (as the source approaches the detector, the number of detected particles will increase, while the number of emitted particles will remain the same). The spectrometer with the HPGe detector was calibrated according to the detection efficiency using the set of reference spectrometric gamma-sources, which included the following isotopes: ${ }^{60} \mathrm{Co},{ }^{133} \mathrm{Ba},{ }^{137} \mathrm{Cs},{ }^{241} \mathrm{Am}$, and ${ }^{22} \mathrm{Na}$. In view of the above, we can conclude that it is possible to calculate the activity of any isotope by the absolute method (according to the number of detected quanta), only knowing the value of the absolute detector efficiency for quanta of a given energy. However, if the sample under study differs geometrically from the reference one, the activity cannot be calculated in this way (the absolute efficiency of the plate and the point source will differ). In this regard, in gamma radiation spectrometry, the concept of geometric efficiency is introduced (Bushuev et al. 2016) as the ratio of the number of detected particles to the number that fell into the volume of the detector $N_{h i t}$ :

$$
\varepsilon_{\text {geom }}=\frac{N_{\text {det }}}{N_{\text {hit }}}
$$

This parameter determines only the property of the detector substance itself to detect gamma quanta of certain energy and is related to the absolute efficiency by the following expression:

$$
\varepsilon_{\text {geom }}=\frac{N_{\text {det }}}{N_{\text {hit }}}=\frac{N_{\text {det }}}{G_{g} \cdot N_{\text {emit }}}=\frac{\varepsilon_{a b s}}{G_{g}},
$$

where $G_{g}$ is the geometric factor, which is a part of the solid angle at which the detector is seen from the point of the source location. In view of the above, the activity of a sample, the geometry of which differs from the reference one, and the half-life of the target isotope is significantly 


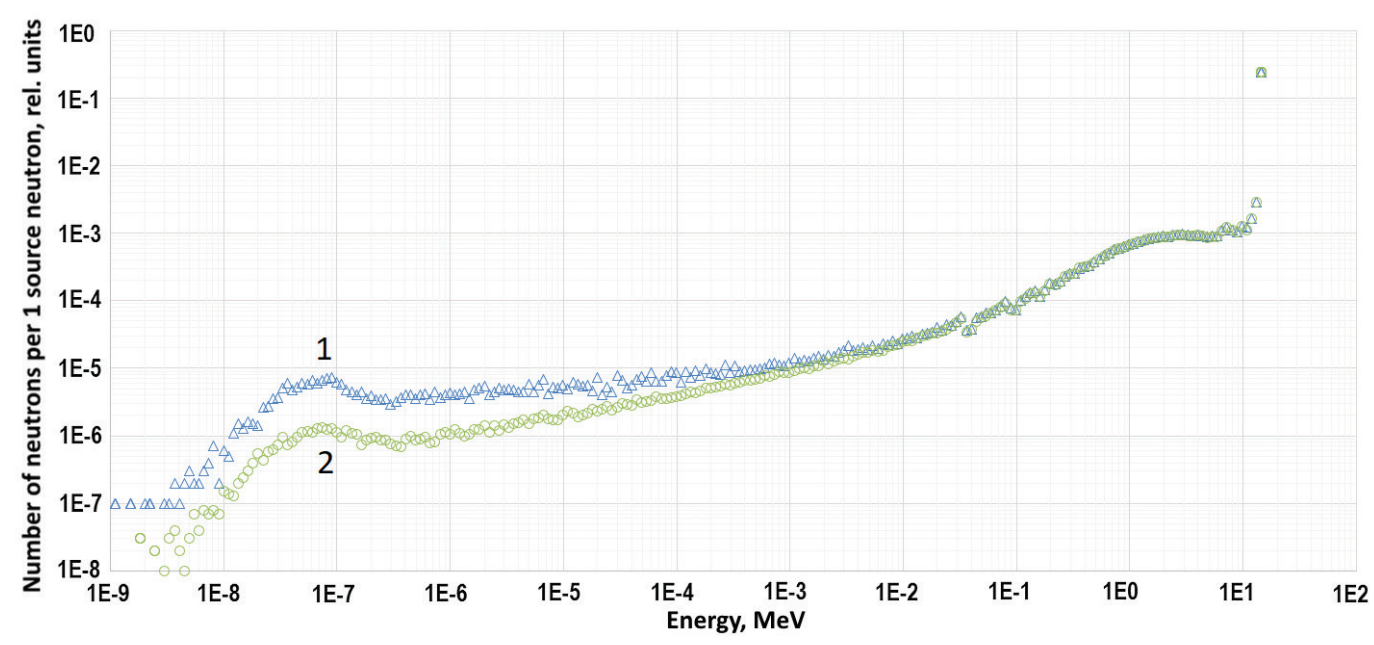

Figure 3. The neutron spectrum obtained using Geant 4 at the location of the activation detectors from a monoenergetic point isotropic source of $14 \mathrm{MeV}$ neutrons - the generator target: 1 - with biological protection made of borated polyethylene $50 \mathrm{~cm}$ thick; 2 - without protection.

longer than the measurement time $t$, can be calculated using the following formula:

$$
A=\frac{N_{\mathrm{det}}}{G_{g} \cdot \varepsilon_{\mathrm{geom}} \cdot I \cdot t},
$$

If the half-life of the isotope under consideration is comparable in magnitude with the measurement time $t$, formula (5) takes the form

$$
A_{\text {start meas. }}=\frac{\lambda \cdot N_{\text {det }}}{1 \cdot G_{g} \cdot \varepsilon_{\text {geom }} \cdot\left(1-e^{-\lambda \cdot t}\right)},
$$

where $A_{\text {start meas. }}$ the activity of the target isotope at the start of the recording of the gamma radiation spectrum; $\lambda$ is the decay constant of the target isotope. At the same time, the activity at the beginning of the measurement is related to the activity at the end of the irradiation $A_{\text {finish exp. }}$ through the law of radioactive decay, taking into account the time elapsed between the end of irradiation and the beginning of measurement of $t_{\text {delay }}$ :

$$
A_{\text {finish exp. }}=A_{\text {start meas. }} \times \exp \left(\lambda \times t_{\text {delay }}\right) .
$$

This value can be linked to the number of neutrons $\Phi$ of a certain energy emitted from the source (the neutron generator target) per unit time:

$A_{\text {finish exp. }}=\Phi \times G_{n} \times\left[1-\exp \left(-\rho \times N_{\mathrm{A}} \times \sigma \times c \times d / M\right)\right] \times\left[1-\exp \left(-\lambda \times t_{\text {delay }}\right)\right]$.

In this expression, $N_{\mathrm{A}}$ is the Avogadro constant; $G_{n}$ is the geometric factor 'neutron source - activation detector', which, multiplied by the value of the neutron yield from the source $\Phi$ (neutron/s), gives the number of neutrons hitting the sample; $\rho$ is the sample density, $\mathrm{g} / \mathrm{cm}^{3}$; $\sigma$ is the microscopic section of the considered reaction, $\mathrm{cm}^{2} ; c$ is the concentration of the considered isotope in the sample, rel. units.; $d$ is the sample thickness, $\mathrm{cm} ; t_{i r r}$ is the irradiation time; $M$ is the mole weight, $\mathrm{g} / \mathrm{mole}$. The value in the first square brackets takes into account the fact that the sample has a certain finite thickness and, in our case, is not thin. In most applications that use activation detectors, work is carried out in intense neutron fields, where thin foils are sufficient to obtain the required activity in the sample. In this case, expression (7) is simplified (instead of the first parentheses, the factor remains, which is a positive exponent). Note that the accuracy of $G_{n}$ is determined by the details of the information on the structure of the target unit of the neutron generator tube, and in this work, the $G_{n}$ parameter could be determined only with a significant error, since the characteristics of the generator design are a commercial secret of the manufacturer.

Combining (6)-(8), we obtain an expression for determining the neutron yield from the neutron generator target

$$
\Phi=\frac{\lambda \cdot N_{d e t} \cdot e^{\lambda \cdot t} \text { dela } y}{I \cdot G_{g} \cdot \varepsilon_{\text {geom }} \cdot\left(1-e^{-\lambda \cdot t}\right) \cdot G_{n} \cdot\left(1-e^{-\frac{\rho \cdot N_{a} \cdot \sigma \cdot c \cdot d}{M}}\right) \cdot\left(1-e^{-\lambda \cdot t_{i r r}}\right)},
$$

Geometric factors are the only quantities that can be difficult to calculate. Their values can be calculated in various ways. For example, in the case of point geometry, the 'activation detector - secondary radiation detector', $G_{\mathrm{g}}$ can be calculated by the following expression:

$$
G_{g}=\frac{1}{2} \int_{0}^{\theta_{2}} \sin \theta d \theta=\frac{1}{2}\left(1-\cos \theta_{2}\right)=\frac{1}{2}\left(1-h / \sqrt{h^{2}+r^{2}}\right),
$$

Figure 4 explains the values given in expression (10).

The shaded area in the figure represents the sensitive volume of the detector made in the form of a cylinder with height $d$ and radius $r$. The distance between the top end of the detector and the point where the source is located is $h$. Expression (10) is valid when the geometric factor is calculated for point sources (sources can be considered as such if their linear dimensions are more than several times smaller than the characteristic dimensions of the detector 


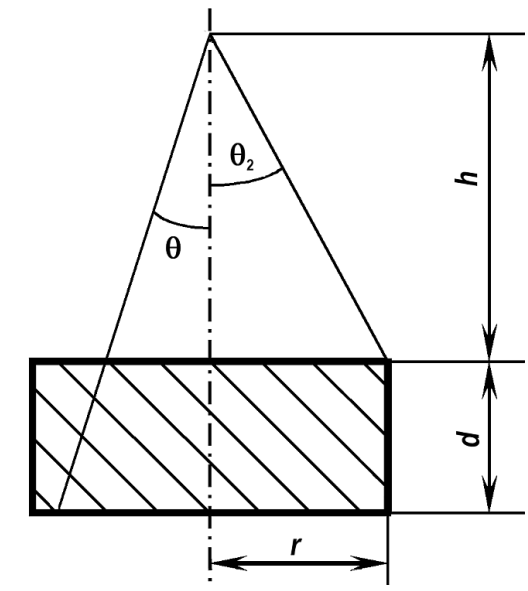

Figure 4. Drawing for determining the value of the geometric factor $G_{\mathrm{g}}$ in point geometry.

and the distance to it). The geometrical factor calculated in this way for the configuration of the experiment with reference spectrometric gamma-sources was $(1.07 \pm 0.02) \cdot 10^{-3}$.

In addition to this analytical method, the Monte Carlo method can also be used for calculating the geometric factor, i.e., randomly playing the directions of rays emitted from each point of the source and fixing the number of their hits on the detector surface. The value of $G_{\mathrm{g}}$, calculated using Geant4, where modeling is based on the Monte Carlo method, turned out to be $(1.06 \pm 0.01) \cdot 10^{-3}$. Modern means of modeling interactions between ionizing radiation and matter make it possible not only to calculate the geometric factors $G_{\mathrm{g}}$ and $G_{n}$, but also to do it, taking into account the possible absorption or other attenuation of both gamma and neutron radiation in structural materials (the housing of the detector or neutron generator, the activation detector substance itself, etc.). For this, it is necessary to specify the composition of materials as accurately as possible. In this regard, in equations (7) and (9), there are no correction factors that should take these processes into account. In addition, using Geant4, it is possible to calculate the theoretical value of the activity of the irradiated sample (or the rate of production of the target isotope) based on the available information on the parameters of the experiment on irradiation of the samples with neutrons.

Since the studied activation detectors are geometrically very different from the point reference sources, which were used for calibrating the gamma radiation spectrometer in terms of efficiency, it was necessary to pass from absolute to geometric efficiency. The transition was carried out according to formula (4) using the values of $G_{\mathrm{g}}$ calculated by means of the Geant 4 tools.

It is necessary to note the point on the obtained dependence (Fig. 5), which is associated with the detected line of gamma quanta with energies of $511 \mathrm{keV}$ emitted by ${ }^{22} \mathrm{Na}$. These gamma quanta are formed as a result of acts of positron annihilation, the source of which is this isotope. In its usual form, the source of reference spectrometric gamma-sources, being an active substance enclosed between two thin polyamide films, does not prevent the emission

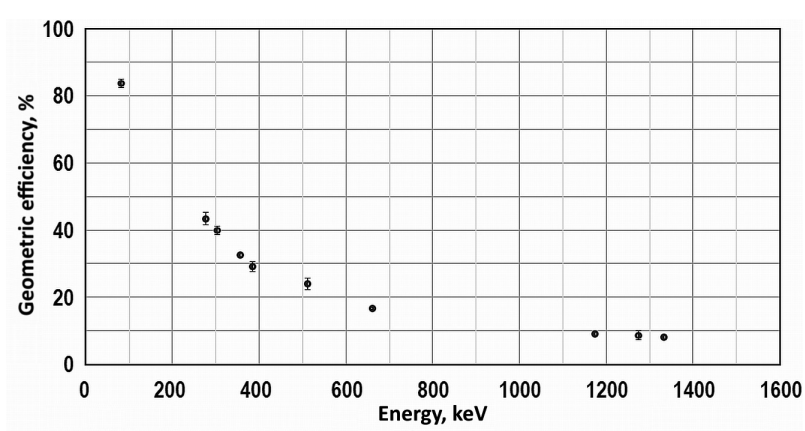

Figure 5. Dependence of the geometric efficiency of detecting gamma quanta on their energy for the HPGe detector (from the experimental facility).

of positrons, which annihilate only at a certain distance from the active substance. Thus, this source can no longer be considered a point source. We can assume that gamma quanta are emitted from a certain sphere with a radius equal to the most probable range of positrons in air. For the source to be considered as a point source, when gamma quanta with energies of $511 \mathrm{keV}$ are detected, it is necessary to place a thin layer of metal foil under and above the source, in which annihilation will occur.

The neutron irradiation of the $\mathrm{Al}$ and $\mathrm{Cu}$ samples was carried out for 20 and $10 \mathrm{~min}$, respectively. Control data on the neutron yield from the generator target during irradiation were taken from the ING-07T control program. Each sample was irradiated at the control yield value of $\Phi_{\text {contr }}=1,5 \cdot 10^{8} \mathrm{n} / \mathrm{s}$, which, taking into account the geometry of the experiment, formed the flux density of fast neutrons at the point of location of the activation detectors of the order of $10^{6} \mathrm{n} / \mathrm{cm}^{2} \cdot \mathrm{s}$, which is a small value compared to flux density values in most experiments with activation detectors. As a result of processing the gamma-radiation spectra, after irradiation of the investigated detectors, the values of the activity of accumulated nuclides at the time of the beginning of the gamma-spectrum recording were obtained. Geant 4 was also used for calculating the theoretical activity value for the described experimental parameters. Table 1 summarizes the results obtained.

The values of the neutron yield from the source can be obtained from the activity values. To calculate the value of the neutron yield from the generator target, it is necessary to make the assumption that we know the most probable energy of the detected neutrons. Taking into account the value of the accelerating voltage set on the neutron generator during the experiment on the irradiation of the sample, it can be assumed that the most probable energy of neutrons emitted from the target was about $14 \mathrm{MeV}$. Note that, in this case, the scatter of possible values of the cross-section of the mentioned reaction is from 450 to $500 \mathrm{mb}$. Table 2 summarizes the results of determining the neutron yield from the ING-07T target using various activation detectors, with errors accounted for. The main contribution to the error in the neutron yield calculated using formula (9) is made by the following parameters (the relative contribution of each parameter to the final relative error is indicated in parentheses): 
Table 1. Values of $G_{\mathrm{g}}$ and $G_{n}$ found using Geant4 and comparison of the values of the induced activity obtained in the experiment $A_{\text {exp }}$ and calculated in the model $A_{\text {model }}$.

\begin{tabular}{|c|c|c|c|c|}
\hline Sample & $G_{g}$, rel. units & $G_{n}$, rel. units & $A_{\text {exp }}, \mathrm{Bq}$ & $A_{\text {model }}, \mathrm{Bq}$ \\
\hline$\overline{\mathrm{Cu}}$ & $(1.21 \pm 0.02) \cdot 10^{-2}$ & $(0.221 \pm 0,011) \cdot 10^{-2}$ & $(5.1 \pm 0,1) \cdot 10^{2}$ & $(5.4 \pm 0.1) \cdot 10^{2}$ \\
\hline $\mathrm{Al}$ & $(1.15 \pm 0.02) \cdot 10^{-2}$ & $(0.267 \pm 0,014) \cdot 10^{-2}$ & $(1.2 \pm 0,2) \cdot 10^{2}$ & $(1.4 \pm 0.3) \cdot 10^{2}$ \\
\hline
\end{tabular}

Table 2. Neutron yield measurements.

\begin{tabular}{lccc}
\hline Detector & Reaction & $\begin{array}{c}\text { Experimental } \\
\text { yield, } \mathbf{n} / \mathbf{s}\end{array}$ & $\begin{array}{c}\text { Yield according } \\
\text { to program } \\
\text { indications, } \mathbf{n} / \mathbf{s}\end{array}$ \\
\hline $\mathrm{Cu}$ & ${ }^{63} \mathrm{Cu}(\mathrm{n}, 2 \mathrm{n}){ }^{62} \mathrm{Cu}$ & $(1.45 \pm 0.15) \cdot 10^{8}$ & $1.5 \cdot 10^{8}$ \\
$\mathrm{Al}$ & ${ }^{27} \mathrm{Al}(\mathrm{n}, \mathrm{p}){ }^{27} \mathrm{Mg}$ & $(1.33 \pm 0.13) \cdot 10^{8}$ & \\
\hline
\end{tabular}

- the cross-section of the considered reaction for neutrons of a given energy $(\sim 50 \%)$;

- the geometric factor "neutron source - activation detector' $(\sim 20 \%)$;

- the number of counts at the peak of the total absorption of the gamma line of the isotope formed under the influence of neutrons in the considered activation detector $(\sim 15 \%)$; and

- the detection efficiency of the considered line of gamma radiation $(\sim 15 \%)$.

The rest of the values have small errors, since they are either tabular or measured with good accuracy during the experiment (half-life, molar mass, isotope content in the sample, sample size, etc.).

We consider it necessary to note once again that the results obtained were close to each other and to the data of the ING control program only due to the assumption of the energy spectrum at the generator outlet. If at least the approximate energy distribution of neutrons at their detection point is unknown, then a certain averaged value will have to be taken as the cross-section value, which will lead to a scatter of the neutron yield from the target by tens and hundreds of percent.

An analysis of the results obtained shows good agreement between the calculated and experimental data, which confirms the legitimacy of using Geant4 for calculating geometric factors in the problems of determining the activity of samples with complex geometry (other than point geometry). The main sources of the formation of errors when calculating the induced activity in the experiment or with the help of Geant 4 can be attributed to the inaccuracy of information about the geometry of the experiment as well as the evaluated nuclear data that are used by Geant 4 .

\section{Acknowledgments}

The authors wish to express their gratitude to the staff of the Federal State Unitary Enterprise Dukhov Automatics Research Institute (VNIIA) for the opportunity to conduct experiments using the ING-07T portable neutron generator.

\section{References}

- Allison J [Geant4 Collaboration] (2016) Recent developments in Geant4 Nuclear Instruments and Methods in Physics Research Section A: Accelerators, Spectrometers, Detectors and Associated Equipment 835: 186-225. https://doi.org/10.1016/j. nima.2016.06.125

- Andrukhovich SK, Marcinkevich BA, Hilmanovich AM (2010) The use of short-lived radionuclides for real-time reconstruction of the neutron spectrum of nuclear physics facilities. Vestnik Natsionalnoy Akademii Nauk Belorussii [Bulletin of the National Academy of Sciences of Belarus] 3: 110-118. [in Russian]

- Bushuev AV, Kozshin AF, Aleeva TB (2016) Practical spectrometry of nuclear radiation. Tutorial. NRNU MEPhI Publ., Moscow, 260 pp. [in Russian]

- Jungmin Jo (2018) Initial result of neutron energy spectrum reconstruction using multi-foil activation method in KSTAR. Fusion Engineering and Design 136: 793-796. https://doi.org/10.1016/j.fusengdes.2018.04.010

- Lebaron-Jacobs Laurence, Gaillard-Lecanu E, Briot F (2008) Dosimetric management during a criticality accident. Nuclear Technology 161: 27-34. https://doi.org/10.13182/NT08-A3910

- Medkour Ishak-Boushaki G (2012) Fast neutron spectrometry using thick threshold detectors. EPJ Web of Conferences 24: 1-9. https:// doi.org/10.1051/epjconf/20122407009

- Neutron Generators (2020) Neutron generators for elemental analysis of substances and materials. http://www.vniia.ru/production/incl/ prospekt_element.pdf [accessed Jan 15, 2020]

- Noba CR (2019) Neutron spectrum unfolding for the development of a novel neutron detector for fusion. Fusion Engineering and Design 146: 2658-2662. https://doi.org/10.1016/j.fusengdes.2019.04.074

- Pierre C, Gilles G, Guillaume Rousseau, Xavier Jacquet, Nicolas Authier (2016) Characterization of the CALIBAN Critical Assembly Neutron Spectra using Several Adjustment Methods Based on Activation Foils Measurement EPJ Web of Conferences 106: 1-8. https://doi.org/10.1051/epjconf/201610607005

- Uddin MS, Zulquarnain MA, Qaim SM (2013) Fast neutron spectrum unfolding of a TRIGA Mark II reactor and measurement of spectrum-averaged cross sections. Radiochimca Acta 101: 613-620.

- Vagena E (2018) Thick-foils activation technique for neutron spectrum unfolding with the MINUIT routine - Comparison with GEANT4 simulations. Nuclear Inst. and Methods in Physics Research, A, 887: 64-69. https://doi.org/10.1016/j.nima.2018.01.025 